\title{
Coronavirus Disease 2019 Coexistence in the Daily Practice
}

\author{
Marco Tallarico ${ }^{1, \odot}$ Marco Cicciù ${ }^{2, \odot} \quad$ Aurea Immacolata Lumbau ${ }^{1} \quad$ Silvio Mario Meloni ${ }^{1}$
}

\author{
${ }^{1}$ Department of Medical, Surgical and Experimental Sciences, \\ University of Sassari, Sassari, Italy \\ ${ }^{2}$ Department of Biomedical and Dental Sciences, Morphological \\ and Functional Images, School of Dentistry, University of Messina, \\ Policlinico G. Martino, Via Consolare Valeria, Messina, Italy
}

\begin{abstract}
Address for correspondence Marco Tallarico, Department of Medical, Surgical and Experimental Sciences, University of Sassari, Sassari 07100, Italy (e-mail: me@studiomarcotallarico.it).
\end{abstract}

\begin{abstract}
Keywords

- COVID-19

- Public Health

Dentistry

- Infection control, dental

- Primary prevention
\end{abstract}

\section{Introduction}

The coronavirus (CoVs) are RNA viruses characterized by the presence of 80 to $160 \mathrm{nM}$ crown-shaped peplomers with positive polarity. The other feature is its high mutation rate that constantly develops transcription errors, as well as RNA replicase (RNA dependent RNA Polymerase, RdRP) jumps. These features make CoVs zoonotic pathogens with a wide range of clinical features from asymptomatic to severe infections, most of which acute respiratory syndrome (SARS), requiring hospitalization in the intensive care unit. ${ }^{1,2}$

First cases of SARS from CoVs have been seen in China, state of Guangdong, in the 2002 and 2003. About 10 years later, CoVs-related Middle East respiratory syndrome (MERS) has been observed. ${ }^{3}$ In our time, novel coronavirus has been detected on December 12, 2019 in China, the Wuhan State of the Hubei Province, which caused global pandemic. ${ }^{4}$ The CoVs are common in many different animal

DoI https://doi.org/

$10.1055 / \mathrm{s}-0040-1719217$

ISSN 1305-7456. species, including camels, cattle, cats, and bats. Wild animal trade in the Huanan Seafoods Market has been known as the origin area. ${ }^{5}$ At first, it was an unknown pneumonia case with fever and other coronaviruses-related symptoms. Nevertheless, about 1 month later (January 7, 2020), Chinese authorities announced that a new CoV was isolated, ${ }^{5}$ then named COVID-19 (World Health Organization [WHO]). The outbreak of the new coronavirus has been declared a pandemic global emergency by the WHO (March 11, 2020), and global quarantine by $\mathrm{CoV}$ spread as the main factors to control the disease. At the time of writing this manuscript, 22,705,645 cases of COVID-19 have been reported, including 794,104 deaths (https://www.ecdc. europa.eu/en/geographical-distribution-2019-ncov-cases). There is an important difference between affected countries mostly because different case definitions and testing strategies were applied. Moreover, people suspected of having COVID-19 may be higher than the official total, since some

\footnotetext{
(c) 2020. European Journal of Dentistry.
}

This is an open access article published by Thieme under the terms of the Creative Commons Attribution-NonDerivative-NonCommercial-License, permitting copying and reproduction so long as the original work is given appropriate credit. Contents may not be used for commercial purposes, or adapted, remixed, transformed or built upon. (https://creativecommons.org/licenses/by-nc-nd/4.0/)

Thieme Medical and Scientific Publishers Pvt. Ltd., A-12, 2nd Floor, Sector 2, Noida-201301 UP, India 
mild and asymptomatic cases are not tested and counted; as a consequence, the number of infected people increases every day. The lockdown contributed to contain the spread of the virus. Nevertheless, the number of new COVID-19 cases has risen sharply over the past 2 weeks. So the question is what happens next? For this reason, to prevent CoV spread and the consequent "second wave," all the people should be considered infected and general rules must to be strictly maintained, including safe and healthful social and working conditions. According to the occupational risk pyramid for COVID-19 published by the Occupational Safety and Health Administration (OSHA, osha.gov/covid-19), dentist is classified as a job with very high exposure risk to known or suspected sources of COVID-19. First, dentists need intimate contacts (within 3 feet) with people, and then dental health care workers performed every day during dental work, aerosol-generating and blood-borne procedures from potentially infected patients. The human transmission of $\mathrm{CoV}$ occurs with close contact with one another through the respiratory droplets produced by infected people, mostly when person coughs and sneezes. These droplets can settle down in the mouth or nasal mucosa of people who are nearby (within about 3 feet) and/or potentially inhabited into the lungs breathing with. ${ }^{6,7}$ On March 15, 2020, the New York Times published an article entitled "The Workers Who Face the Greatest Coronavirus Risk." For this reason, primary concern of dental health care workers is the own infection prevention and control measures against the new coronavirus, adopting proper and certified personal protective equipment (PPE). However, for the same principle, the second concern is that respiratory droplets can land onto the nearly surfaces. Currently, it remains unclear but very possible that a person can be infected by COVID-19 by touching an infected surface or object and then touching their mouth, nose, or possibly eyes. ${ }^{7-9}$ While it should be easy to protect itself from infection, this scenario expose at high risk of cross infection and subsequently spread of the infection within patients, practitioner, and person in contact with all of them, such us parents and family. ${ }^{8,9}$

Dentistry is essential health care because of its role in evaluating, diagnosing, preventing, or treating oral diseases, which can affect systemic health. Nevertheless, because of the infection risks associated with aerosol-generated procedures, dentists around the world have been essentially closed since late March 2020. During this period, there was limited access to emergency dental care. Dental services are now slowly and tentatively beginning to reopen, although there is considerable variation in the guidance being issued on the safety procedures required. As governments mandates lift and dentists reopen practices, this narrative review does not want to be a standard of care or official regulation, and it does not create legal obligations. This manuscript presents recommendations of mandatory and safety dental health care standards, intended to help dentists in providing a safe and healthful workplace during pandemic, that means live together with the COVID-19. The main object of this narrative review was to evaluate and discuss the actual available information regarding COVID-19 and dental practice to answer to these questions: how can dental health care workers protect itself from $\mathrm{CoV}$ infection? And how can dentist reduce the risks of cross infection in their clinics?

\section{Patients and Methods}

The present narrative review was conducted following current data and previously published practical guidelines on COVID19. The focused question was: COVID-19 coexistence in the daily practice. A literature search of available manuscripts was performed to identify relevant studies according to the focused question. The PubMed database of the U.S. National Library of Medicine and relevant websites ( - Supplementary Material [available in online version]) have been consulted. A critical analysis of the available literature was performed by the authors and grouped in five sections.

\section{Measures to Reduce Surfaces Contamination before Dental Treatments}

Prevention plays an important role in protection from the disease and to reduce risk of cross infection. ${ }^{10,11}$ Of course, dental health care workers must consider all the potential infected people. Nevertheless, typically, like most respiratory viruses, people are thought to be most contagious when they are most symptomatic. Phone coronavirus triage is mandatory to evaluate risk level of our patients. The survey must be done the day of the visit and it begins by asking patients if they are currently experiencing:

- Severe cough, shortness of breath, fever, or other concerning symptoms

- Contact with confirmed case(s) of COVID-19

- Quarantine or isolation due to suspect or conclamate COVID-19

- Vulnerable patients (>60 years and/or patient with concomitant pathologies) should postpone the therapy if possible

- Any other known reasons that may expose patients to be a high risk of spread of the infection

Before the sch eduled appointment, the secretary must contact the patient asking to answer the questionnaire and reminding her/him to follow some general rules. Vice versa, the secretary must reassure the patient that the dental clinic is a safe place where all the recommendations to contain infection are strictly adhered to.

- To arrive in the dental clinic alone or with maximum one companion if needed (i.e., child treatment), avoiding assembly of people

- To leave in the waiting room potentially infected objects (jacket, bag, phone, etc.)

- To accurately hands cleaning at the arrival or to wear gloves

- To wear the face mask up to dental chair

- To rinse the mouth for 3 minutes with alcohol-based mouthwash before treatment 
Further considerations will regard the availability of a rapid serological blood-based test that should be able to identify whether people have been exposed to COVID-19.

\section{Measures to Reduce Degree of Contamination during Dental Treatments}

It should be better to postpone all the "not necessary therapies" to "better days." The authors really understand that most of the dental procedure should be the matter of urgency even if not symptomatic or paucisymptomatic. The American Dental Association classified dental emergency in urgent and not urgent therapies (https://www.ada.org/en). For the latter, not urgent therapies are initial or periodic oral examinations, recall visits, routine dental cleaning and preventive therapies, orthodontic procedures, extraction restorative dentistry of asymptomatic teeth, and aesthetic dental procedures.

To define and limit the risk areas, the authors proposed to identify three areas of risk ( $\boldsymbol{- \text { Fig. }} \mathbf{1}$ ). The dental unit represents a "high-risk zone" (or red zone) due to surfaces contamination and generation of aerosol during most of the dental treatments. This area has a radius of approximately 3 feet from the patient's mouth. Outside this area, all the operative room, including adjacent working areas (such as dental laboratory, sterilization room, dental office, patient toilette, etc.) must be considered a "medium-risk zone" (orange zone) due to possible contamination of the surfaces. These areas could be contaminated directly by touching the surfaces with gloves during the dental treatments by the main operator, dental assistant, patient, or indirectly, bringing infected material. All the rest of the dental clinics must be considered a "low-risk zone" (yellow zone) because it is not possible to exclude possible contamination. Red zone required high level of protection and attention.

- Use rubber dam when possible or other specific tools to improve dental isolation (e.g., specially designed mouth retractors) and to reduce aerosol generation (e.g., specially designed suction evacuator).

- Encourage use of speed-increasing handpiece instead of turbine handpiece

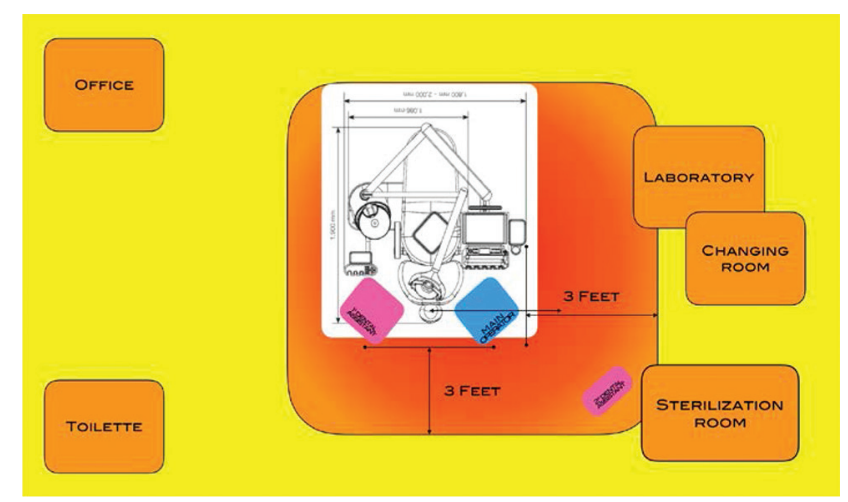

Fig. 1 Identification of risk zones. Red zone includes dental chair and main operators. This is the higher risk zone due to air (aerosol) and surface contamination. Orange zone is a medium risk due to high possibility or surface contamination. Yellow zone represents all the dental clinic where possible surfaces contamination could not be completely excluded.
- Encourage manual scaling instead of ultrasonic teeth cleaning during professional dental hygiene procedure

- Avoid dental treatments with airflow

- Encourage use of digital impression with intraoral scanner to avoid use of impression materials, reducing risk of cross infection. ${ }^{12}$ Furthermore, to avoid dental laboratory delivery of potentially infected materials, even if decontamination procedures are performed according to the actual rules.

- Self-contained water system should be used instead of current water to supply water to handpieces (including external piezo ultrasonic scalers) and syringes. This allows for adding sterile hypochlorous acid for sanitizing the water. Hypochlorous acid is safe and approved by the Food and Drug Administration. Contact times of 1 minute or greater are typically sufficient to achieve a thorough kill including viruses. The concentration of sanitizer in the wash water must not exceed 200 ppm hypochlorite. The patient must be rinsed with potable water following the chlorine use.

- Apply a team-based chain of control that means organizing a logical workplace zones and operative sequences. At least two dental assistants should assist the main operator. The first, if required, besides the operator to offer direct assistance. The second, external dental assistant, with the purpose to avoid external contamination outside the "high-risk zone" that consists of a perimeter line separated at least 3 feet from the main operator and patient's mouth.

\section{Personal Measures to Protect Dental Healthcare Workers}

Dentists and coworkers (dental assistant, collaborators, partitioners, etc.) have always used PPE. Nevertheless, due to the nature of the new CoV and different risks of transmission, adjunctive PPE must to be integrated in daily practice along with those already used. In this context, the authors consider the main operator (dentist or dental hygienist) the worker that perform the dental procedure. The operator can work alone or he/she may need of assistance by a dental assistant (or nurse). Whenever possible, the operation should work alone in the "high-risk zone." Nevertheless, it should not improve own risks or risk for cross infections.

According to the Center for Control Diseases, $\mathrm{N}-95$ respirators without exhalation valve or surgical masks must be used. The closest European equivalent to N95 are the filtering face piece (FFP2) face mask. Both are suitable for CoVs disease prevention and are suggested for dental health care workers. The main differences between this PPE is that the edges of the mask are not designed to form a seal around the nose and mouth. So with surgical mask is mandatory a full covered face shield. ${ }^{9}$ Dentists that use dental loupes must wear N95 (or FFP2) mask. Dental loupes should allow eye protection. Nevertheless, companies are producing specially designed face shield to be used in conjunction with dental loupes. Same products:

- Dental protective face shield designed to be easily decontaminated with alcohol or immersion disinfection contributes to protect the mouth and noise from aerosol, as well 
as other exposed mucous membranes, which includes the eyes. The face shield should have at least two protective transparent shield (one is for substituted) and cover all the face including lateral portion.

- Disposable full-length gown made of fluid-resistant material.

- Gloves have always played a very important role in the disease prevention in medicine, but there is "little widespread evidence" that they are useful for members of the public. Dental health care workers must wear gloves as usual. It is authors' opinion that there is no need to wear a double pair of gloves in the daily practice for personal protection. Nevertheless, to reduce possible cross infection, a second pair of gloves could help dental health care workers to reduce risk of contamination during disposable PPE discharging (layered undressing).

- A head covering that provides an effective barrier is recommended during any procedure that is likely to result in the splattering of blood or aerosol. It is authors' opinion that shoe covering are not mandatory to reduce the spread of CoV. Nevertheless, workers can use it if they want.

- To maintain the greatest possible distance, dental assistant should assist while standing.

- Main operator must have maximum care during prosthetic procedures that may require adjustment in adjacent room (i.e., dental laboratory). If possible he/she should delegate this procedure, avoiding PPE discharging and wearing.

Most of disposable PPE are designed for single-use and must be accurately discarded after every patient. For example, according to the manufacturer some face mask could be used up to 8 hours (if not directly contaminated). Others could be sterilized up to 200 times. It is most important to carefully read and follow manufacture instruction.

Work uniforms cannot be taken at home. They must to be washed in office following specific programs, or better yet, handled by a dedicate external laundry that also provided pickup and delivery service.

Further considerations regard the availability of a serological test able to measure the amount of antibodies against COVID-19. This test should be performed regularly to eventually make an early diagnosis of exposed workers. This allows for early care of the worker, but also preventing the spread of the virus.

\section{Measures to Reduce Risks of Cross Infection after Patient Treatment}

All the dental clinics must establish a proper protocol including a strictly workflow to manage patient discharging and dental room cleaning and decontamination before treating next patient. This includes higher time compared with pre-COVID-19 scenario. Dental clinics must consider that in this period, overall number of patients per day must to be reduced allowing for more time to establish proper procedures before and after dental treatments.

After dental procedure, all the disposable PPE must carefully discard, starting to help patients to properly leave the dental chair.
Then, all the main operators must properly wash their hands, arms, and face. At this point, the dentists may bring the patient to the office (or away from the clinic), while both assistants take care of the dental room cleaning and sterilization.

All the surfaces in the "high-risk zone" must be carefully and properly decontaminated, including not disposable PPE. External assistant must control all the used materials and instruments taking care to reduce as soon as possible risks of contamination during and between dental procedures. It is of importance that external assistant clearly establishes the perimeter of the "high-risk zone" and also she/he takes care to avoid contamination outside this area. She/he also must control possible contamination outside the "high-risk zone" during dental treatment and provide best decontamination accordingly (moving to dental laboratory for adjustments).

It is important to respect the time needed to kill or inactivate microorganisms. Dental clinics must use proper disinfectants certified for CoVs and respect its protocol.

During dental room cleaning and sterilization, the assistants must to wear PPE.

Frequent hand washing with proper soap is needed during all of these phases.

Regarding sterilization procedures, at the time of writing this narrative review, there is no evidence that adjunctive procedures are needed. Dental clinics should be already organized with high-quality instruments for the decontamination process in practice and clinic.

There is no evidence about patient discharge/management at the office level. The secretary must use same PPE as dental operator, limited to face mask and gloves, always maintaining social distance of at least 3 feet. In addition, some companies are providing plastic face screen to install to the desktop for operator and patient protection. It's authors' opinion that after dental procedure the patients could be contaminated form own aerosol generated during dental procedure. To reduce possible contamination, next appointments and payments could be manage as follows:

- Next appointment and payment could be managed before dental procedure.

- Electronic payment should be preferred.

- If a companion is present, he/she can manage appointment and payment.

- Next appointment and payment could be managed by phone at later stage.

- All the desktop and potentially contaminated surfaces must be carefully decontaminated.

\section{General Recommendations for the Dental Healthcare Workers and the Public}

Promote frequent and thorough hand washing with proper soap, including by providing workers, customers, and worksite visitors with a place to wash their hands.

Encourage workers to stay home if they are sick (fever, cough, and difficulty breathing) and seek medical care early as follows: 
- Practice respiratory hygiene encouraging respiratory etiquette, including covering coughs and sneezes.

- Maintain social distancing when possible, avoiding assembly of people through a well-organized rescheduled program of the appointments.

- Avoid touching eyes, nose, and mouth.

- Carry put cleaning and decontamination of the used surfaces outside the dental room (i.e., keyboard, mouse, pen, desks, tables, telephones, etc.).

- Stay informed and follow advice given by your health care provider

\section{Discussion}

At today more than 22 million cases have been confirmed and they are likely to increase, so pandemic is far to disappear. The WHO expects 2 years for the end of the pandemic, assuming a fast availability of an effective vaccines. To make this situation worse, recently, most of the infected people were asymptomatic or paucisymptomatic, and their viral load seems to be improved.

As aforementioned explained, the objective of this review article was to share a preliminary opinion about the disease, the possible ways of treat dental patient, and all the effort to prevent dental healthcare workers and patient cross infection in this early stage of COVID-19 outbreak. Concerning our dental clinics and the consolidated use of PPE by dental operators, it is authors' opinion that the main not-clinical problems could be the difficulties to purchase PPE, the unknown patient feel and reaction, and the unavoidable economic crisis. Industry is quickly developing PPE to improve their availability. Regarding patients' feel and economic crisis, at the moment, it is not possible to expect when the COVID-19 pandemic and social distancing will end, and what will be the consequences. People will always need to take care of their teeth, but maybe, there will be some changes in their priority and maybe the need for sustainability treatments. Moreover, a fear of a "second wave" may complicates further scenario.

Regarding the dental-related concerns, it is authors' opinion that the main problems are to ensure the highest level of safety for our staff, patients, and family. In this way, prevention at the beginning and the proper controls of cross infection at the later stage are the main important area to improve in our workflows.

Routine cleaning of the workplace, without additional disinfection is recommended at this time. Particular attention to clean the frequently touched surfaces, such as workstations, counter-tops, and door handles. The cleaning agents used in a dental workplace are suitable for the disinfection. Nevertheless, the directions on the label must to be adored to. To improve contamination control, disposable wipes should to be placed nearly the commonly used surfaces (e.g., doorknobs, keyboards, remote controls, and desks).

Dentists must provide proper PPE for all the staff, and update the document of risk assessment, including all the procedures needed to reduce overall risks, as explained in this document. The deleted appointments should be reschedule giving a list of priority to reduce the number of patients per day, starting from urgent therapy. This may help dental assistant to perform all the needed procures to clean and decontaminate the potentially contaminated surfaces.

Due to the characteristics of dental workplace, the risk of cross infections may be high be-tween dental healthcare workers and patients, mainly due to aerosol-generating procedures. ${ }^{10-15}$ Prevention still remains the first and most important step to reduce risk that potentially infected people coming in the clinic. Within this, telephone triage could be an important starting point for future perspectives, such as telemedicine and virtual patient management. ${ }^{16}$ Then a well-established chain of control has been proposed to reduce risk of contamination before during and after the treatment. Dentists and dental assistants already use PPE during their daily practice. ${ }^{11-13}$ Nevertheless all the staff, including, not health workers must to be trained for PPE use. Moreover, protection from a virus is a challenge because it is not possible to see it. In this context, definition of different risk zones could help dental healthcare workers to manage all the procedures act to protect from and prevent cross infections. The red zone required high level of personal protection and accurate decontamination of the surfaces. The orange zone required general personal protection and accurate decontamination of the surfaces. All the dental clinic should be considered potential source of contamination due to public place, so it is not possible to exclude possible contamination. For the latter, yellow zone only required general rules, such us social distance, frequent, and thorough hand washing as well as avoid touching eyes, nose, and mouth.

Human-to-human transmissions have been described with incubation times between 3 and 14 days via droplets, contaminated hands, or surfaces. According to the WHO seems that the COVID-19 is mainly transmitted through contact with respiratory droplets rather than through the air. Nevertheless, waiting consistent data, it is supposed that CoVs could persist on inanimate surfaces form 72 hours up to 9 days, but they can be efficiently inactivated by surface disinfection procedures. ${ }^{14,15}$ Adopting the proper operative workflow, management of the risk zones and generals rules, including standard ventilation, extensive decontamination procedures at the end of a daily work seem not to be justified.

People can be infected everywhere, including dental clinic. Nevertheless, at the date of writing this manuscript, any outbreaks in a dental clinic was noted. The higher risks seem to be in restaurants, beaches, logistics, disco, and nursing homes. Moreover, many of Italy's recent confirmed cases of COVID-19 are from Italians traveling back from high-risk zones to their homes. Compulsory swabs for travelers as well as serological blood-based test for workers could be very helpful to evaluate quarantine in case of infection and to potentially identified infected patients before treatment.

Finally, the COVID-19 global pandemic continues to have devastating health, economic, and social effects, and dentistry is not excluded. However, rather than resuming normal service, this crisis presents an opportunity to rethink the future of dentistry and address system-level failures, as an 
opportunity to improve. For instance, dental professional contributed to create printed plastic valves to mitigate the shortage of respiratory devices during the COVID-19 epidemic by adapting the dental digital workflow and converting snorkeling masks in emergency "continuous positive airways pressure" devices. ${ }^{17}$

\section{Summary}

The present narrative review offers deeper knowledges and discussion regarding recommended procedures aimed to reduce risk for dental healthcare workers contamination and cross infection. Dental office prevention of coronavirus infection, including triage, respect of general rules, containment measures, proper use of the PPE, and well-organized chain control of all the staff, with definition of risk zones and specific responsibility still remain the most important steps to control the spread of the disease.

\section{Authors' Contributions}

M.T. and M.C. helped in conceptualization. M.T. supported in methodology. S.M.M. provided resources. M.T. dedicated in writing original draft. M.A.L. and S.M.M. contributed in writing and review and editing. M.C. offered supervision.

\section{Funding}

This work was supported by funding by University of Sassari (to L.A.I.) (grant number: FAR2019).

\section{Conflict of Interest}

None declared.

\section{References}

1 Drexler JF, Gloza-Rausch F, Glende J, et al. Genomic characterization of severe acute respiratory syndrome-related coronavirus in European bats and classification of coronaviruses based on partial RNA-dependent RNA polymerase gene sequences. J Virol 2010;84(21):11336-11349

2 Yin Y, Wunderink RG. MERS, SARS and other coronaviruses as causes of pneumonia. Respirology 2018;23(2):130-137

3 Zaki AM, van Boheemen S, BestebroerTM, Osterhaus AD, Fouchier RA. Isolation of a novel coronavirus from a man with pneumonia in Saudi Arabia. N Engl J Med 2012;367(19):1814-1820

4 Seven days in medicine: 8-14 Jan 2020. BMJ 2020;368. doi:10.1136/bmj.m132
5 Meng L, Hua F, Bian Z. Coronavirus disease 2019 (COVID-19): emerging and future challenges for dental and oral medicine. J Dent Res 2020;99(5):481-487

6 Peng X, Xu X, Li Y, Cheng L, Zhou X, Ren B. Transmission routes of 2019-nCoV and controls in dental practice. Int J Oral Sci 2020;12(1):9

7 Sahin AR, Erdogan A, Mutlu Agaoglu P, et al. 2019 Novel Coronavirus (CO- VID-19) Outbreak: A Review of the Current Literature. EJMO 2020;4(1):1-7

8 WHO. Emergencies preparedness, response. Pneumonia of origin - China. Disease outbreak news. Available at: www.who. int/csr/don/12-january-2020-novel-coronavirus-china/en/. Accessed February 5, 2020

9 Rothe C, Schunk M, Sothmann P, et al. Transmission of 2019-nCoV Infection from an Asymptomatic Contact in Germany. N Engl J Med 2020;382(10):970-971

10 Barenghi L, Barenghi A, Cadeo C, Di Blasio A. Innovation by computer-aided design/computer-aided manufacturing technology: A look at infection prevention in dental settings. Biomed Res Int 2019;2019:6092018 doi:10.1155/2019/6092018

11 Barenghi L. News from the COVID-19 front in an Italian Dental Practice. EC Dental Sci-ence 2020;19(4):157-161

12 Sabino-Silva R, Jardim ACG, Siqueira WL. Coronavirus COVID-19 impacts to dentistry and potential salivary diagnosis. Clin Oral Investig 2020;24(4):1619-1621

13 Spagnuolo G, De Vito D, Rengo S, Tatullo M. COVID-19 outbreak: an overview on Dentistry. Int J Environ Res Public Health 2020;17(6):2094

14 Cervino G, Fiorillo L, Surace G, et al. SARS-CoV-2 persistence: Data summary up to Q2 2020. Data 2020;5:81 doi:10.3390/ data5030081

15 D'Amico C, Bocchieri S, Stefano R, et al. Dental office prevention of coronavirus infection. Eur J Dent 2020;14(suppl S1): S146-S151 doi:10.1055/s-0040-1715923

16 Cervino G, Oteri G. COVID-19 pandemic and telephone triage before attending medical office: problem or opportunity? Medicina (Kaunas) 2020;56(5):250

17 Cavallo L, Marcianò A, Cicciù M, Oteri G. 3D printing beyond dentistry during COVID 19 epidemic: a technical note for producing connectors to breathing devices. Prosthesis 2020;2:46-52 